\title{
The Exploitation of Microcomputer Processing System to the Meteorological Satellite Images Based on the Remote Sensing Physical Technology

\author{
Gaofeng Pan ${ }^{1, \text { a }}$, Chuyan $\mathrm{Li}^{1, \mathrm{~b}}$, Ying Zheng ${ }^{1, \mathrm{c}}$ \\ ${ }^{1}$ Department of Aviation Theory, Aviation University of Air Force, Changchun 130022, China \\ aemail:27867752@qq.com, bemail:274402491@qq.com, cemail:273906295@qq.com
}

Keywords: Meteorological satellite images; Remote sensing physics technology; Microcomputer processing system

\begin{abstract}
Meteorological satellite images are very important in meteorology, and they have a clear reflection to the process of the weather's dynamic changes. Remote sensing technology can give the detection and identification to the objective, and it has the effective identification to the weather pictures. In this paper, the innovation is the application of remote sensing technology to the processing of meteorological satellite images, and it has achieved good results that getting the high recognition rate and the clear transmission data. On this basis, the author develops a set of microcomputer processing system to the satellite images using the remote sensing physics technology.
\end{abstract}

\section{Introduction}

As people's requirement on the weather and the meteorological service quality is increasing, the traditional meteorological service cannot gradually meet their needs. Meteorological satellite images are playing an increasingly important role in modern meteorology, and the meteorological satellite images with high quality and high resolution reflect accurately the physical process of the meteorological dynamic variation. And in recent years the remote sensing technology is more and more widely applied and popular. Remote sensing physical technology, as a kind of technology used in detection, was beginning in the 1960s, and its principle is based on the theory of electromagnetic wave. It takes the radiation and reflection to the distance object and target using various sensors, and makes the images information through the feedback information and data. Currently every 18 days the man-made satellite can send back a global image data. Using remote sensing technology can survey and map the target with high speed and high quality. In order to further improve the speed of image processing, greatly reduce the cost and improve the popularization of the technology, in this paper the author plans to develop a set of microcomputer system to the meteorological satellite images processing based on the remote sensing physical technology [1].

\section{The Basic Characteristic of the Meteorological Satellite Images}

\subsection{Introduction to the meteorological satellite images}

Meteorological satellite, the man-made satellite making the meteorological observation to the atmosphere, belongs to a kind of special satellite or remote sensing satellite for the earth observation. It has the characteristics like large range, timely, rapid, continuous and full, and it can send the meteorological information such as the cloud atlas to the user on the ground. Meteorological satellite images are like the receiving station that meteorological satellite makes the information such as clouds and meteorological conditions into picture and sends it to the ground. The weather satellite images are shown in picture 1 . 


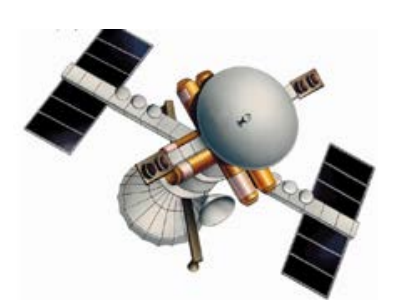

Fig.1. weather satellite images

\subsection{The spread characteristic of the meteorological satellite signal channel}

For meteorological satellite, the precondition of receiving the ground data is that, the ground object remains still and its location is determined, and the characteristics of the surrounding environment is rather open. So some considered factors, which are needed in modeling of the general satellite signal channel, don't need to consider in modeling of meteorological satellite signal channel. According to the meteorological conditions, such as wind, cloud, rain, fog and other comprehensive factors, the author makes the model to the signal channel characteristics of the meteorological satellite [2].

\subsubsection{The effect of multipath propagation}

In the process of filming, the satellite signals will be through the clouds, vapor, mist and other complex meteorological conditions, and through reflection, scattering, diffraction and other variations the strength and direction of the signal transmission will change. When the remote sensing technology is used in meteorological satellite images processing, the decline effect of the small scale must be considered. That effect is the Rice effect and Rayleigh effect [3].

The fog, sand, cloud and other meteorological conditions in the terminal weather don't have the direct effect for the signal. So the receiving signal obeys the Rayleigh distribution, and the amplitude $x$ and phase $\varphi$ obey the following probability density function [4]:

$$
\begin{aligned}
& p(x)=\frac{1}{\sqrt{2 \pi} \sigma} \exp \left(-\frac{x^{2}}{2 \sigma^{2}}\right) \quad 0 \leq x<\infty \\
& p(\varphi)=1 / 2 \pi \quad 0 \leq \varphi \leq 2 \pi
\end{aligned}
$$

If the meteorological condition is poor, and it has the physical phenomena such as reflection and diffraction due to the interference of the clouds and fog, the receiving signal will obey the Rice distribution, and the amplitude $x$ and phase $\varphi$ will obey the following probability density function[5]:

$$
\begin{aligned}
& p(x)=\frac{x}{\sigma^{2}} \exp \left(-\frac{x^{2}+\rho^{2}}{2 \sigma^{2}}\right) I_{0}\left(\frac{x \rho}{\sigma^{2}}\right) ; \quad 0 \leq x<\rho \\
& p(\varphi)=1 / 2 \pi ; \quad 0 \leq \varphi \leq 2 \pi \\
& p(\varphi)=\frac{\exp \left(-\rho^{2} / 2 \pi\right)}{2 \pi}\left\{1+\sqrt{\frac{\pi}{2}} \frac{\rho}{\sigma} \cos \left(\theta_{s}-\varphi\right)^{*}\right. \\
& \left.\exp \left(\frac{\rho^{2} \cos ^{2}\left(\theta_{s}-\varphi\right)}{2 \sigma^{2}}\right)\left[1+\operatorname{erf}\left(\frac{\rho \cos \left(\theta_{s}-\varphi\right)}{\sqrt{2} \sigma}\right)\right]\right\}
\end{aligned}
$$

And in the form $\rho$ is the peak of remote sensing signal; $I_{0}(x)$ is the modified function, $\operatorname{erf}($.$) is$ the error function, and Rice is the variance ratio of the remote sensing main signal and the multipath signal.

\subsubsection{The shadow fading effect of remote sensing physical signal}

In the transmission process of satellite signal, it has the large decline and loss when touching the clouds, and the decline will affect the whole spread path. In the process of shadow fading, the remote sensing physical signals can be described as: 
$p(x)=\frac{1}{\sigma x \sqrt{2 \pi}} \exp \left(-\frac{(\ln x-\mu)^{2}}{2 \sigma^{2}}\right)$

And: $\sigma$ is the variance of meteorological satellite signal and $\mu$ is the mean value of meteorological satellite signal.

In the meteorological satellite signal channel, the multipath propagation characteristic and the shadow fading effect exist at the same time, and according to the weather's condition, it can combine the two kinds of effects to form Suzuki models:

1) When the weather's condition is poor, the formula of the Suzuki models is:

$$
\begin{aligned}
& p(x)=\int_{0}^{\infty} \frac{x}{\sigma^{2}} \exp \left(-x^{2} / 2 \sigma^{2}\right) 1 /\left(\sqrt{2 \pi} \sigma_{s} \sigma\right)^{*} \\
& \exp \left[-\left(\ln \sigma-\mu_{s}\right)^{2} / 2 \sigma_{s}^{2}\right] d \sigma
\end{aligned}
$$

Therefore the model considers fully the two attenuation effects, and it can be suitable for the application that remote sensing physical technology is in the processing of meteorological satellite images[6].

2) When the weather's condition is good, the formula of the Suzuki models is:

$$
\begin{aligned}
& p(x)=\frac{x}{b_{0} \sqrt{2 \pi} d_{0}} \int_{0}^{\infty} \frac{1}{s} \exp \left[-(\ln s-u)^{2} / 2 d_{0}-\right. \\
& \left.\left(x^{2}+s^{2}\right)^{2} / 2 b_{0}\right]^{*} I_{0}\left(x^{*} s / b_{0}\right) d s
\end{aligned}
$$

And: $I_{0}()$ is the modified function of zero order, and $s$ is the direct component of the shadow.

\section{The Development of the Microcomputer Processing System for the Remote Sensing Satellite Images}

This part is mainly about the development of a set of microcomputer system for the satellite images processing based on remote sensing physical technology. It is the first time to make the remote sensing physics technology into the microcomputer system whose characteristics are that improving the resolution of the satellite image processing, reducing the costs and improving the accuracy[7].

\subsection{The design principle}

In order to develop the satellite images processing system which is based on remote sensing technology, this paper chooses the single chip model SDK86, and has the expanded main memory and memory. For obtaining the accurate remote sensing signal and data, it is necessary to ensure the mechanical equipment and CCD physical scanning in sync. The principle diagram of the system is shown in picture 2[8]: 


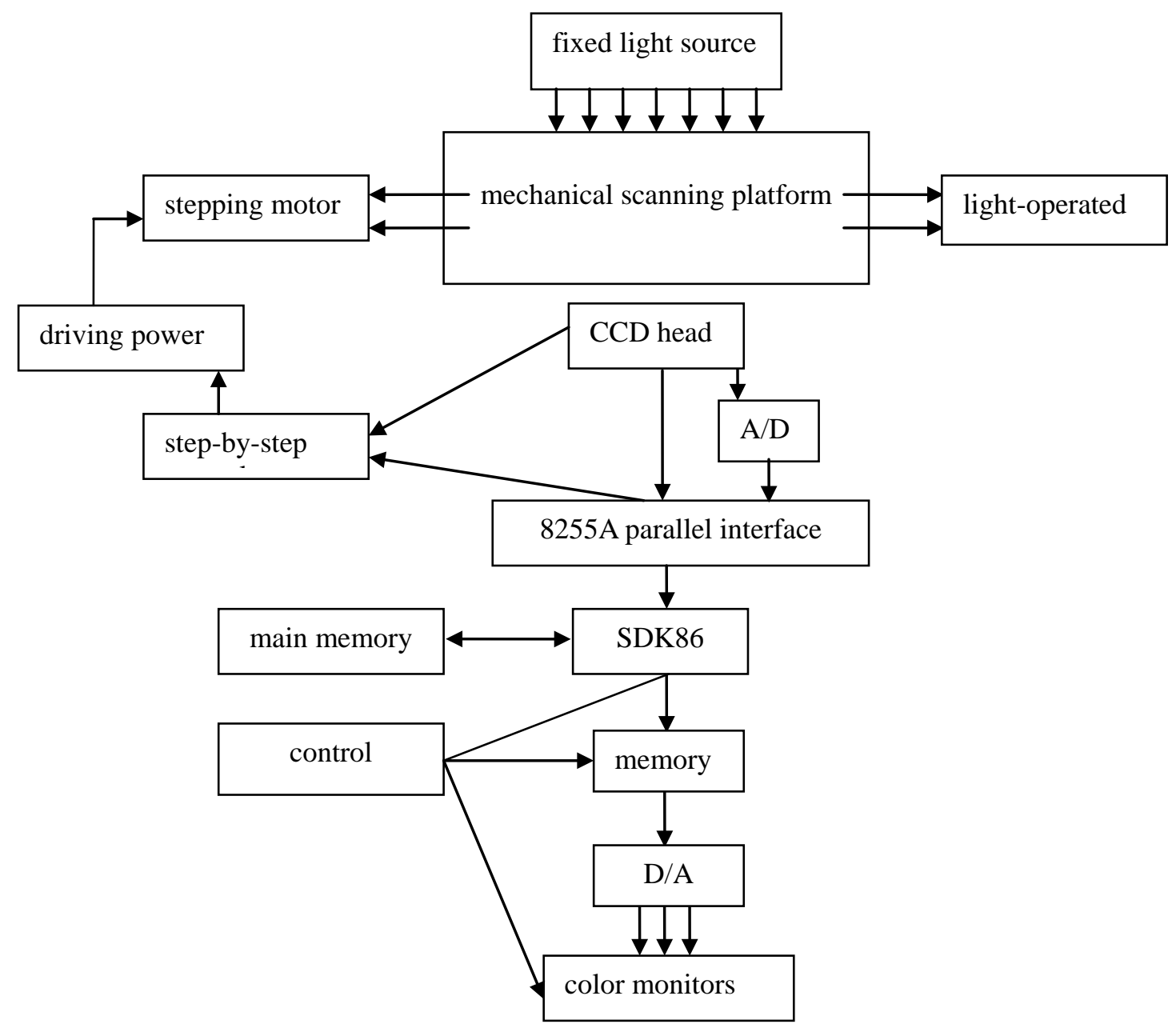

Fig.2. The principle diagram of the system

The system uses a special way, the combination method of CCD linear sweep and mechanical platform translation, to deal with the satellite images. In the $\mathrm{x}$ direction, the scanning adopts the stepper motor to drive the platform to complete the translation[10].

On the control strategy of microcomputer processing system, it uses the 8255A parallel interface in the single-chip SDK86, and uses the photoelectric switch and the drive power to drive. That can realize the functions of the system startup, shut down and the reversing. According to the nature of the meteorological satellite images, the nature of the drive light source can be diversified, and it can choose the perspective light and reflected light at the same time. By adjusting the aperture and focal length of CCD imaging, it makes the imaging of the meteorological satellite images in the CCD effective photosensitive area.

\subsection{The circuit design of the microcomputer processing system}

\subsubsection{The circuit design of the CCD camera}

The linear CCD is the main part in the microcomputer processing system which is based on the remote sensing physical technology for the meteorological satellite images processing, and its principle diagram is shown in picture 3[9]: 


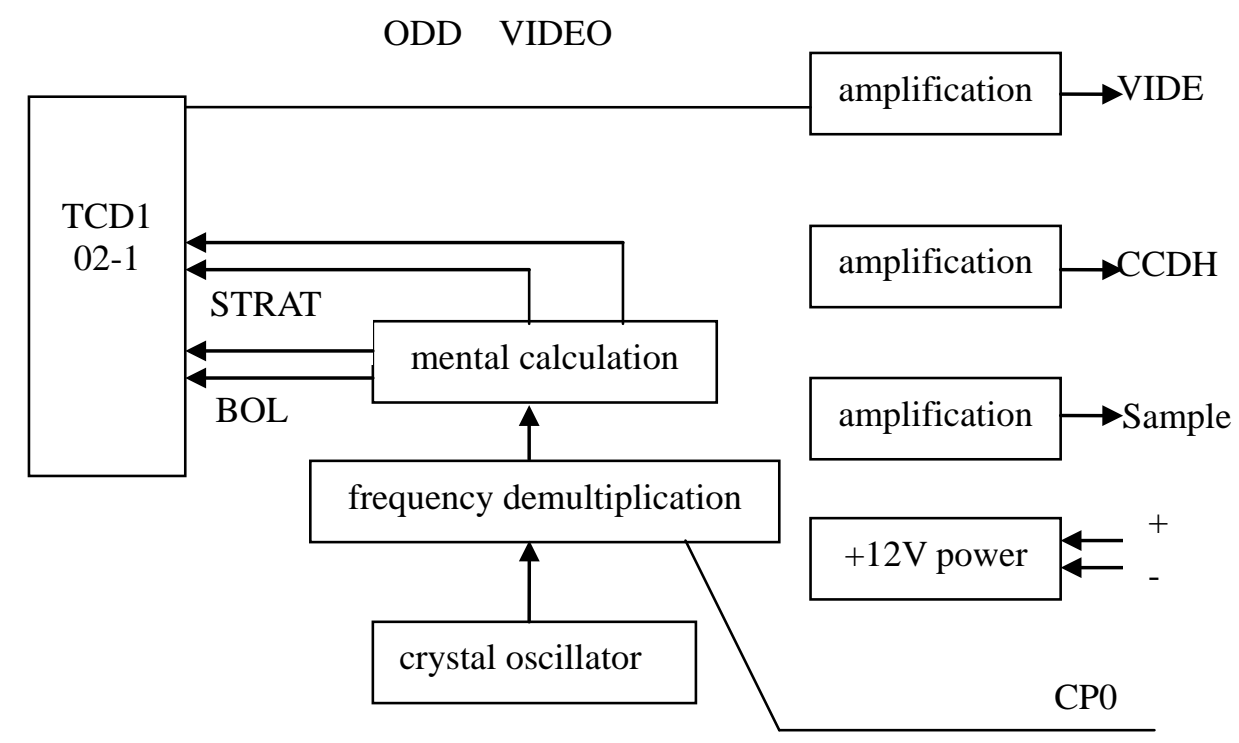

Fig.3. CCD camera principle

\subsubsection{The stepping control and light-operated circuit design}

The driving power adopts the stepper control and photoelectric control to control the motor. For the single chip, there are three interfaces available on 8255 parallel interface. The principle of stepping control and light-operated circuit is shown in picture 4:

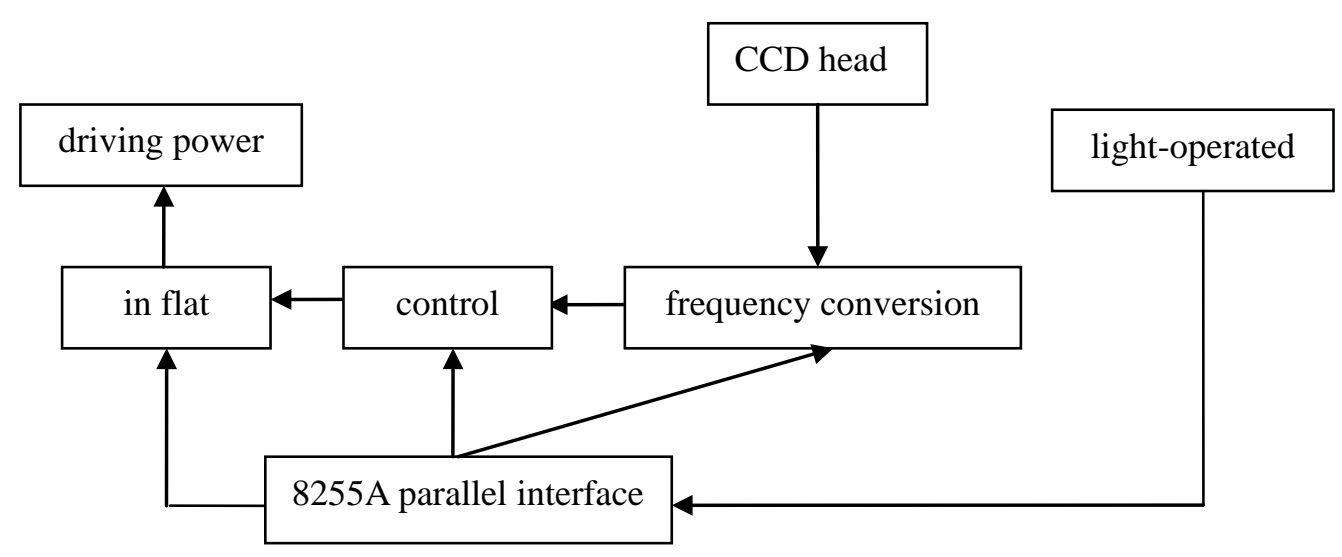

Fig.4. stepping control and light-operated circuit diagram

In the control system of picture 4, the converter stage is transported to the control door by the pulse, and the level transform signal through CT/P is the driving power. The function of 8255A is to make the control signal and driving power signal of the control gate be zero, so through the remote sensing physical technology, the aim is to deal with different sizes of meteorological satellite images.

\subsubsection{The main memory, memory and control circuit}

It is necessary to give the high definition to the meteorological satellite images, so the main memory and memory use the 4164 array. The control signal of main memory is provided by the $64 \mathrm{k}$ dynamic RAM controller 8203, and in the control system it has the buffer effect for the memory system of the meteorological satellite images, which can be controlled by 8203 . The CPU of the single-chip SDK86 can work in the minimizing mode and its clock frequency is about $2.45 \mathrm{MHz}$. In 
the expansion, the main memory and memory of SDK86 single-chip can be decoded by $A_{19}$ and $A_{18}$.

During the processing, the frequency band range of the video signal is wide, whose range is about $6.5 \mathrm{M}$. According to the sampling law, the sampling frequency is 2 times greater than the frequency band range, and its size should be about 13M. But in the selecting process, it generally uses four consecutive pixels to mobile the high pixel data.

The D/A conversion circuit plays a very important role in the control system. It is mainly composed of dynatron, and its ending frequency can reach a high level, so it can meet the operation requirements. After the D/A transformation, the three-channel signal of RGB can be input through the three signal ends of CRT to eliminate the potential between the video signals, so it can realize the purpose and requirements of high definition for the meteorological satellite images.

\section{The Operation and Application of the System}

\subsection{The characteristics of the system}

The system is installed on the system of OLIVETTIM380, and runs through the microcomputer PX5. The hard disk capacity of the system host is about 304MB, and the frequency of the host is about 20MC. In the process of processing data, the system can be divided into four processes: 1 . receive meteorological satellite data through the remote sensing physical technology; 2. do the preprocessing and pretreatment about the collected meteorological satellite data by the microcomputer processing system; 3. do the quantitative processing to the collected information and data; 4. sort, classify and display the processing results. The characteristics of the system has five aspects:

1. The information amount of the system is large, and the data transmission is fast; The resolution ratio of the meteorological satellite images is high, and it is easy to transfer the meteorological satellite images with high resolution ratio when using this set of microcomputer system;

2. The data forms are varied; The processed satellite images information can display through a variety of patterns and means, and the digital signals and physical signals of the satellite images can be switched to each other. And they can be uploaded, downloaded and printed, which is easy for the user and for the research;

3. The system functions is powerful. The system can support the weather forecast, and for the sorting of the meteorological data, mobile phones and the processing of meteorological satellite images with the high resolution, it appears very convenient;

4. It has the high reliability and strong compatibility. The system has the stronger reliability and compatibility. It can analyze and deal with the data of the meteorological satellite images under the different climate conditions, different humidity and different temperature, and its applying range has extend and strengthened in maximum.

\subsection{The processing effect of the meteorological satellite images}

(1) The monorail broadening cloud. In order to eliminate the abnormal of the meteorological satellite images, the system adopts the method of vertex insertion line by line, and makes 2048 scanning spots in a scanning line expand to 3072 scanning spots. Through the geographic latitude and longitude network, it signs the geographic information to get the meteorological satellite images with clearer resolution and more uniform grid distribution. Through the strengthened gray level, the images data of the remote sensing information changes into the monorail broadening cloud with stretched grid distribution and clear resolution. In the range of $4 \mathrm{~km}$, the resolution for this kind of picture can achieve to $512 \times 512$, so in $2 \mathrm{~km}$ range the resolution can be as high as $1024 \times 1024$, and the obtained meteorological satellite images can be used for the analysis of the meteorological weather forecast.

(2) The projection of the puzzle. It can integrate the satellite data in the different orbits, and the 
integrated content is included all kinds of coordinate transformation and geometric correction, etc. The principle is making the different orbital data be together through the certain logic calculation. The image data can reflect the weather characteristics in the large scale environment.

(3) The playback. It can preprocess the meteorological satellite data through the remote sensing physical technology, and the processing result is making the images display and be synthetic, roam and be amplification through the multi-channel colors.

(4) Other images processing. After the preprocessing it continuously makes the images enhance, be amplification, filter and the synthesis of multispectral. Finally it forms the animation display by the calculation of the meteorological regional area to meet the needs of different customers.

\section{References}

[1] Liu H. Y., Cai J. M. Development Document of Meteorological Satellites of Feng Yun Series [J]. Defense Science \& Technology Industry, 2011, 35(6):30-32.

[2] Li J., Fang Z. Y. Opportunities and Challenges of the Development of Meteorological Satellites [J]. Meteorology, 2012, 56(2):129-146.

[3] Liu Y. F. Functions of Cloud Photograph of Meteorological Satellites in Observing Surface Meteorology [J]. Meteorological Hydrological and Marine Instruments, 2011, 24(4):103-105.

[4] Yang G. H., Bai Y. The First Business Type of Earth Relative Rest Meteorological Satellite FY-2C [J]. Meteorology, 2004, 26(12):36-38.

[5] Zhang X. Z., Guo Y. C., Chen J. L., et al. Characteristics of probability statistics of Channels of L \& X Band Meteorological Satellite [J]. Journal of Applied Meteorological Science. 2012, 22(4):478-484.

[6] Zheng X. T., Zhan, Y. J., Zhang Z. N., et al. Analysis and Modeling on Satellite Declining Channels [J]. Electronic Design Engineering. 2011, 96(8):89-91.

[7] Yan Y., Zhang Q. S., Chang Q., et al. Analysis and Realization of Lutz Satellite Channel Model [J]. Journal of Telemetry, Tracking and Command. 2007, 6(6):89-93.

[8] Gao D. B., Dai Y. S., Chen H. X. Research on Statistic Characteristics of Land Moving Satellite Communication Channels [J]. Journal on Communications, 2001, 22(12):117-123.

[9] Yang M. C., Guo Q., Wang Z. Y. Model and Simulation Research on Channels of Land Moving Satellite under the Circumstances of Shadow [J]. Journal of Telemetry, Tracking and Command. 2007, 30(3): 20-24.

[10] Li J. J., Zhang S. Research on Parameter Fitting Method of A New Three-state LMSC Model. [J]. Techniques of Automation and Application, 2010, 35(10):65-68. 